\title{
Document Image Quality Assessment Based on Texture Similarity Index
}

\author{
Alireza Alaei ${ }^{1}$, Donatello Conte ${ }^{2}$, Michael Blumenstein ${ }^{3}$, Romain Raveaux ${ }^{4}$ \\ ${ }^{1,3}$ School of Information and Communication Technology, Griffith University, Australia \\ ${ }^{2,4}$ Laboratoire d'Informatique (LI EA6300), Université François-Rabelais de Tours, France \\ 11alireza20alaei@yahoo.com, ${ }^{3}$ m.blumenstein@griffith.edu.au, ${ }^{2,4}$ \{donatello.conte, romain.raveaux\}@univ-tours.fr
}

\begin{abstract}
In this paper, a full reference document image quality assessment (FR DIQA) method using texture features is proposed. Local binary patterns (LBP) as texture features are extracted at the local and global levels for each image. For each extracted LBP feature set, a similarity measure called the LBP similarity index (LBPSI) is computed. A weighting strategy is further proposed to improve the LBPSI obtained based on local LBP features. The LBPSIs computed for both local and global features are then combined to get the final LBPSI, which also provides the best performance for DIQA. To evaluate the proposed method, two different datasets were used. The first dataset is composed of document images, whereas the second one includes natural scene images. The mean human opinion scores (MHOS) were considered as ground truth for performance evaluation. The results obtained from the proposed LBPSI method indicate a significant improvement in automatically/accurately predicting image quality, especially on the document image-based dataset.
\end{abstract}

Keywords: Document images, Image quality assessment, Texture features, Local binary patterns (LBP).

\section{INTRODUCTION}

With the emergence of new digitization technologies such as smartphone cameras, tablets, portal scanners, and digital cameras, the daily substantial production of images, which is beyond manual processing, is a real challenge. In such a scenario, one of the important issues is the quality of the generated images. As image quality is a subjective characteristic, generally, a human opinion score (HOS) or mean human opinion score (MHOS) needs to be considered to determine the image quality. Concerning the huge volume of different types of images generated in daily life, it is quite obvious that human opinions cannot be considered to assess the quality of such a massive volume of images.

In the past two decades, many image quality assessment (IQA) measures have been developed to automatically provide objective image quality and to handle predicting natural scene image quality. The first attempt dealing with this problem used the simplest mean squared error (MSE) metric, computed by averaging the squared intensity differences of distorted and reference image pixels, along with the related quantity of peak signal-to-noise ratio (PSNR). It has been shown that these metrics are not very well matched to perceived visual quality [1]. Some of the sophisticated IQA models for natural scene images have been presented in [1-6, 18, 19]. Based on the availability of the original/reference images, the IQA methods presented in the literature have been categorized into three main groups of: (i) full reference (FR) [1-4], (ii) no reference (NR) [5-6, 19], and (iii) reduced reference (RR). Most methods [1-4] in the literature are based on the availability of original images, with which the distorted images are to be compared (the socalled FR approach). This kind of approach has further been used as a learning step in the "blind" or "NR" image quality assessment approaches, where no reference image is available [19]. FR IQA methods are mainly based on the assumption that the structural information changes can provide a good approximation to perceived image distortion $[2,4,18]$.

In the domain of document image analysis, as new technologies provide handy facilities for people, a massive volume of different document images is generated day-byday. These documents are not only simple text sheets, they are colorful and stylish with aesthetically pleasing, textured backgrounds and graphical content. Analysis and recognition of such a huge volume of complex document images is time consuming and expensive. Therefore, many methods have been introduced in the literature to automatically predict the quality of document images prior to the application of any further document image analysis process. A high portion of the studies and methods related to the DIQA has considered OCR results as metrics of measuring document quality [7-9]. A brief review of such a category of DIQA methods has been presented in [10].

A few research studies have also been carried out in the domain of document image analysis to develop metrics, which compute automatic objective image quality for document images [11-14]. In these methods, HOSs have been used as ground truth to evaluate how objective image quality computed by a machine is close to the human perception-based image quality scores provided by individuals [11-14]. A FR DIQA method has been presented for binary document images at the character level in [11]. The method is based on a simple distance-reciprocal distortion measure between two pixels. In [12], a system for binary DIQA at the character level based on morphologicalbased features, noise removal-based features and spatial characteristic features has been proposed. A neural network has been trained for estimating qualities of character images [12]. In [13], a DIQA model for document images captured by smart-phones has been proposed using sharpness information. The difference of changes in gray scale values of a median-filtered image has been used as an indication of edge sharpness [13]. Recently, a FR DIQA method based on gradient magnitude features has further been proposed [14] The method (MGMSD) presented in [14] has mainly used 
foreground information as a set of patches extracted from the reference document images employing a segmentation technique. For each foreground patch, the corresponding gradient maps obtained from the reference and distorted gradient magnitude maps have been used to compute a gradient magnitude similarity map of the patch. Gradient magnitude similarity deviation of the patch has then been calculated and an average pooling has finally been performed to obtain the final image quality metric of the distorted document image [14]. In [14], we proved that the MGMSD metric was more effective on document images than some existing measures. We further argued that new kinds of features needed to be investigated in order to improve the performance of DIQA metrics.

Considering the state-of-the-art methods for DIQA using the MHOS for performance evaluation, we noted that a few methods have been proposed for DIQA [13, 14]. Furthermore, document images may contain complex foregrounds/backgrounds and fancy ornamentations that OCRs fail to provide good recognition results for in spite of their good quality. In addition, document image acquisition, compression, and transmission methods deal mainly with color/gray document images. Therefore, the development of automatic approaches for predicting document image quality correlating with MHOS is in high demand.

In this research work, a novel FR DIQA measure based on texture features is proposed. Texture information, as an important feature, has been used for the analysis of various types of images in different applications [15, 16]. Local binary patterns (LBP), as a specific type of texture feature, have been proven to be very powerful for texture analysis and classification $[15,16]$. The LBP method is gray scale invariant [15] and can be computed in terms of an LBP map and LBP histogram. In our proposed method, initially, some preprocessing techniques such as color to gray conversion, filtering, and down-sampling are employed to enhance the image quality, and prepare both reference document images and their distorted versions for further processing. LBP maps and LBP histograms as local and global features of both reference and distorted document images are obtained. For each extracted LBP feature set, a similarity measure/index called an LBP similarity index (LBPSI) is computed. A weighting strategy is further proposed to improve the LBPSI obtained based on the local LBP features. The LBPSIs computed for both local and global features are then combined to obtain the final LBPSI as a document image quality metric, which also provides the best performance for DIQA. The contributions presented in this research work can be noted as: i) exploring the use of LBP-based features at two levels for DIQA, ii) integrating a weighting strategy based on gradient magnitude in the proposed LBPSI method for DIQA, iii) proposing a DIQA metric called LBPSI based on the combination of local and global features.

The remainder of this paper is laid out as follows: Section II describes our proposed FR DIQA method. Section III illustrates the experiments, results and comparative analysis. Finally, Section IV concludes the paper providing future perspectives for the work.

\section{PROPOSED FR DIQA METHOD}

To facilitate a quick overview of our proposed FR DIQA method and its steps in this research work, a block diagram of the proposed method is presented in Fig. 1. Details of each step are described in the following subsections.

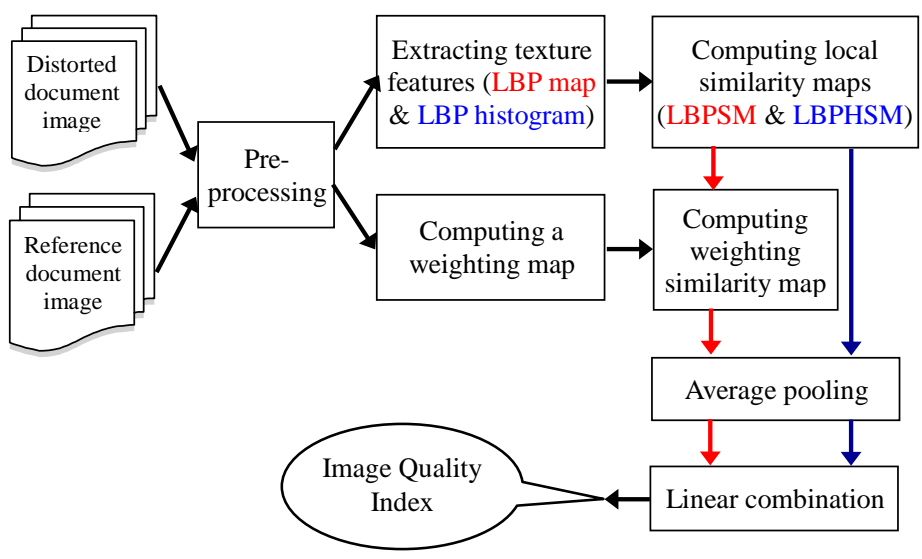

Fig. 1. Block diagram of the proposed FR DIQA method (LBPSI).

\section{A. Pre-processing}

In the pre-processing step of the proposed FR DIQA method, both the reference and distorted document images are converted into gray-scale images. Then both gray-scale images are subjected to a mean filtering process to smooth the document images [4, 14]. Since, down-sampling has provided better performance in a shorter execution time in many IQA models, such as the SSIM [2], FSIM [3], GMSD [4], and MGMSD [14], down-sampling is further performed on both filtered document images.

\section{B. Computing local LBP similarity maps}

A similarity measure has been used for predicting image quality in many IQA methods such as SSIM [1, 2], FSIM [3], GMSD [4], and MGMSD [14]. In all similarity-based approaches for IQA, a similarity value has commonly been computed for every pixel of the image based on different features/characteristics extracted for that pixel. The average pooling has further been used to obtain final quality of the image.

To characterize every pixel in an image, different features such as gradient magnitude [3, 4, 14], luminance [2], contrast [2], structural features [2] and pixel intensity have been used in different state-of-the-art methods for IQA. Texture features and especially LBP as a texture feature have been used in the literature in different applications such as texture classification, face recognition, signature verification and leaf classification [16]. The LBP provides a robust way for describing different textures of various types of images. A brief description of computing the basic LBP is presented in the following. Readers can refer to $[15,16]$ for further details. In the basic LBP [15], every block of $3 \times 3$ extracted from the original image (Fig. 2a) is thresholded by the value of the center pixel in that block to obtain a binary $(0 / 1)$ pattern. The binary values of the pixels in the thresholded block (Fig. 2b) are multiplied by the weights given in the corresponding pixels (Fig. 2c) to obtain decimal values as 
shown in Fig. 2d. Summation of the values of the eight pixels is then considered as the LBP of this block (texture unit) [15]. Since, the block size (texture unit) is considered as a $3 \times 3$ pixel size, the LBP map of an image of size $M \times N$ becomes $(M-2) \times(N-2)$ in size. That means for all the pixels located in the border of the image, LBP values are not computed. As all the values in the LBP map are between 0 and 255, a histogram of the values is also computed providing a 256 LBP histogram feature vector for an image. By computing the occurrence histogram, structural and statistical concepts are effectively combined. The LBP map detects microstructures, such as edges, lines, spots and flat areas, whose underlying distribution is estimated by the LBP histogram [15].

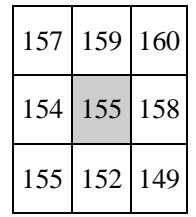

(a)

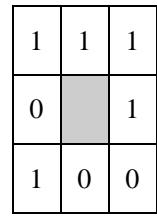

(b)

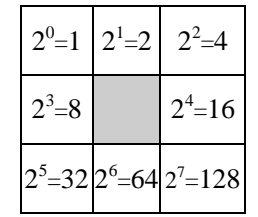

(c)

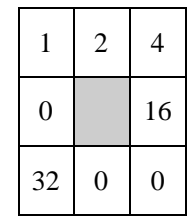

(d)
Fig. 2. Computing the LBP features [15]: (a) a texture unit (block of size $3 \times 3$ pixels), (b) binary pattern obtained based on thresholding, (c) corresponding weights for pixels, and (d) result obtained from the multiplication of (b) and (c).

As in this research work a FR DIQA method is proposed, LBP maps and LBP histograms for both reference and distorted document images are computed. The LBP map represents local information, since, the values in the LBP map are computed for each pixel of the image considering only neighboring pixels. Whereas, the LBP histogram provide a global representation of the image, as it provides a compact description of the image. To formularize the problem, let $R_{M, N}$ and $D_{M, N}$ be a reference document image and a distorted version of the reference image with a size of $M \times N$ pixels, respectively. The LBP maps, which are called $L B P M R$ and $L B P M D$, are computed for both $R_{M, N}$ and $D_{M, N}$ employing the following formulas.

$$
\begin{aligned}
& \operatorname{LBPMR_{M-2,N-2}}=\operatorname{LBPF}\left(R_{M, N}\right) \\
& \operatorname{LBPM} D_{M-2, N-2}=\operatorname{LBPF}\left(D_{M, N}\right)
\end{aligned}
$$

where $L B P F$ is the function which constructs the LBP map based on the abovementioned procedure shown in Fig. 2.

Considering the $L B P M R$ and $L B P M D$ computed for the reference and the distorted images, a LBP similarity map (LBPSM) is computed as the local quality map of the distorted image $D$. The $L B P S M$ is defined as follows:

$$
L B P S M_{M-2, N-2}=\frac{2 \times L B P M R_{M-2, N-2} \times L B P M D_{M-2, N-2}+c}{L B P M R_{M-2, N-2}^{2}+L B P M D_{M-2, N-2}^{2}+c}
$$

where $c$ is a small constant value used to ensure a stable result for the $\operatorname{LBPSM}[3,4,15]$. The optimum value of $c$ is defined in Section III.

As a compact (global) texture feature set of size 256 features, histograms of LBP, which are called $L B P H R$ and $L B P H D$ are also computed for $R_{M, N}$ and $D_{M, N}$ to further characterize the reference and distorted images as follows:

$$
\begin{gathered}
\operatorname{LBPHR}_{1, V}=\operatorname{LBPHF}\left(R_{M, N}\right) \\
\operatorname{LBPHD_{1,V}}=\operatorname{LBPHF}\left(D_{M, N}\right)
\end{gathered}
$$

where $L B P H F$ is the function which generates a LBP histogram as mentioned in [15] and $V=256$ is the size of global histogram feature vector.

With regards to the $L B P H R$ and $L B P H D$ computed for the reference and the distorted images, a LBP histogram similarity map ( $L B P H S M$ ), similar to the LBPSM (3), is also computed as a quality map of the distorted image $D$. The $L B P H S M$ is based on global LBP histogram information. The $L B P H S M$ is defined as follows:

$$
\operatorname{LBPHSM}_{1, V}=\frac{2 \times L B P H R_{1, V} \times L B P H D_{1, V}+c}{L B P H R_{1, V}^{2}+L B P H D_{1, V}^{2}+c}
$$

As a result, two similarity maps (LBPSM, LBPHSM) based on local (LBP map) and global (LBP histogram) information are computed to be later used in order to predict the quality of the distorted document image $D$.

\section{Computing weighting map and quality index}

Average pooling, as the simplest way of computing the quality score, has frequently been used to obtain the final quality score for an image from its local quality/similarity map. A more sophisticated pooling strategy called weighted pooling has also been used for computing the final quality of an image in the literature $[1,3,18]$.

In this research work, the average pooling method is initially employed on both similarity maps (LBPSM, LBPHSM) extracted based on LBP features to demonstrate the behavior of the LBP-based features regarding two different perspectives (local and global representation). The average similarity, so called local binary pattern similarity index (LBPSI), is separately computed for the LBPSM and $L B P H S M$ using the following equations:

$$
\begin{array}{r}
L B P S I M=\frac{1}{(M-2) \times(N-2)} \sum_{m=1}^{M-2} \sum_{n=1}^{N-2} L_{n}{ }_{\text {PPS }}, n \\
L B P S I H=\frac{1}{V} \sum_{i=1}^{V} \text { LBPHSM }_{1, i}
\end{array}
$$

To improve the proposed $L B P S I$ with regards to the local features (LBPSIM), we further introduced a weighting strategy based on gradient magnitude information to be integrated in equation (7). In many state-of-the-art methods, gradient magnitude features have been used for FR IQA [3, $4,14,18]$. In this research work, gradient magnitude features extracted from both filtered reference and distorted images are used to create a weighting map. As mentioned, the LBP map detects microstructures, such as edges, lines, spots and flat areas in images. Gradient magnitude also signifies the edge pixels of images. Therefore, the integration of gradient features as a weighting map to the LBP similarity map influences the structure of edges, lines and spots, which carry important information in all images especially document images. To obtain the document image gradient, a Prewitt filter with a $3 \times 3$ template gradient is employed $[4,14]$. The Prewitt filters in the $X$ and $Y$ directions are defined as $F_{X}$ and $F_{Y}$. The gradient magnitudes of $R_{M, N}$ and $D_{M, N}$ referred to as $G R_{M, N}$ and $G D_{M, N}$ are computed employing the following equations. 


$$
\begin{aligned}
G R_{M, N} & =\sqrt{\left(R \otimes F_{X}\right)^{2}+\left(R \otimes F_{Y}\right)^{2}} \\
G D_{M, N} & =\sqrt{\left(D \otimes F_{X}\right)^{2}+\left(D \otimes F_{Y}\right)^{2}}
\end{aligned}
$$

where the sign $\otimes$ is the convolution operation. Considering the gradient magnitudes $G R_{M, N}$ and $G D_{M, N}$ computed for reference and distorted images, a weighting map called $W$ is computed as:

$$
W_{M, N}=\frac{2 \times G R_{M, N} \times G D_{M, N}+t}{G R_{M, N}^{2}+G D_{M, N}^{2}+t}
$$

where $t$ is a constant used to obtain a stable result for $W$.

The LBPSM obtained based on equation (7) is then multiplied cell by cell with the computed gradient magnitude based weighting map $(W)$. The average pooling is then employed to obtain the weighted LBP similarity index (LBPSIWM). To formularize the abovementioned explanation for computing weighted LBPSI, the following equation is produced:

$$
\text { LBPSIWM }=\frac{1}{(M-2) \times(N-2)} \sum_{m=1}^{M-2} \sum_{n=1}^{N-2} W_{m+1, n+1} \times L B P S M_{m, n}
$$

Considering the equations (7), (8) and (12), we may note that in all the three equations, information extracted from either LBP maps (local information) or LBP histograms (global information) are used for predicting document image quality. To use both local and global information extracted based on LBP texture, both similarity indices extracted from the weighted LBP map (LBPSIWM) and the LBP histogram $(L B P S I H)$ are linearly combined to compute the final local binary pattern similarity index (LBPSI). The combination of $L B P S I H$ and $L B P S I W M$ is presented by:

$$
\begin{gathered}
L B P S I=\alpha \times L B P S I H+\beta \times L B P S I W M \\
\beta=1-\alpha
\end{gathered}
$$

As shown in equation (14), $\alpha$ and $\beta$ are two real values between 0 and 1 , and each one can be computed based on the other one.

\section{EXPERIMENTAL RESULTS AND DISCUSSION}

\section{A. Datasets and evaluation protocol}

We considered two different datasets to conduct different experiments for evaluation of the proposed method. The first dataset is the ITESOFT dataset [14], which includes 377 document images of which 29 are reference images and the rest (348 images) are distorted document images. The reference document images have been collected from real world data using different capturing devices such as a mobile camera, steel camera, and scanner. JPEG and JPEG2000 at six different levels have been applied on reference images to generate distorted images. MHOS has been provided for each document image based on the HOSs collected from 23 individuals. We asked individuals to score each image based on the quality of the image and its readability. Individuals used 19-inch monitors with a distance 40-50 cm from their faces for image quality assessment. The second dataset, which is called the LIVE [17] dataset, is a collection of natural scene images used widely for evaluation of IQA methods. The LIVE is mainly used for comparison of the results with some state-of-the-art metrics. Some statistics about these two datasets are shown in Table I.

Following the tradition in natural scene IQA in general, and DIQA in particular, the Root Mean Square Error (RMSE), the Pearson Linear Correlation Coefficient (PCC) and the Spearman Rank Order Correlation Coefficient (SRC) were used to evaluate the performance of the proposed LBPSI method and to compare it to some state-of-the-art methods. RMSE, PCC and SRC were computed based on the results provided by the LBPSI method and the MHOSs provided for images of each dataset as ground truth. PCC measures the degree of linear relationships between two variables. SRC measures how well the relationship between two variables can be described using a monotonic function. The value of RMSE should be close to 0 whereas values of PCC and SRC should be near to 1 for better performance and efficiency of any IQA method.

TABLE I. SOME STATISTICS OF THE DATASETS USED FOR EXPERIMENTATION.

\begin{tabular}{lccccc}
\hline \hline Database & $\begin{array}{c}\text { No. of } \\
\text { Reference } \\
\text { Images }\end{array}$ & $\begin{array}{c}\text { No. of } \\
\text { Distorted } \\
\text { Images }\end{array}$ & $\begin{array}{c}\text { No. of } \\
\text { Distortion } \\
\text { Types }\end{array}$ & $\begin{array}{c}\text { Type of } \\
\text { Images }\end{array}$ & $\begin{array}{c}\text { No. of } \\
\text { Observers }\end{array}$ \\
\hline ITESOFT [14] & 29 & 348 & 2 & Document/Color & 23 \\
LIVE [17] & 29 & 779 & 5 & Scene/Color & 161
\end{tabular}

\section{B. Implementation and parameter settings}

In the proposed LBPSI technique, there are three parameters, which need to be set. The first parameter is the value of $c$ used in equations (3) and (6). As discussed earlier, $C$ should be a small value to obtain stable results for the LBPSIM and LBPSIH. We considered different values for $c$ and computed the results. From the results obtained, we noted that using values smaller than 0.01 for the parameter $c$ provided stable results on the ITESOFT dataset. Hence, $c$ was set to 0.0001 . The same process employed to fix the parameter $c$ was performed to set the parameter $t$. We found that values greater than 150 provided stable results for LBPSIWM. Therefore, $t$ was set to 170 as in $[4,14]$. The last parameter is $\alpha$, which needs to be adjusted. We experimentally set $\alpha$ to 0.3 . A detailed discussion of the settings for those parameters is provided in the next subsection. A Matlab implementation of the proposed system will be publicly available for research purposes.

\section{Results and discussion}

The performance of the proposed LBPSI method was evaluated considering the ITESOFT dataset [14]. As the proposed LBPSI demonstrated in equation (13) is composed of different elementary metrics, the results obtained for each element of the LBPSI metric and final LBPSI results are presented in Table II. From the results shown in Table II, it is clear that those obtained based on the similarity map computed concerning the LBP map (LBPSIM) are lower than the other results. It conveys that the use of only local features by the means of a LBP map is not good enough to accurately predict the quality of document images. In contrast, using the LBP histogram, as global information for predicting document image quality in the $L B P S I H$ metric, provided reasonably better results compared to the local LBP 
map features used in the LBPSIM metric. The results obtained based on the weighted LBPSI (LBPSIWM) showed $3.1 \%$ improvement $(0.031=0.919-0.888)$ in terms of the PCC with respect to the results achieved using only local LBP map features in the LBPSIM metric. It proves the assumption that integrating weights into the local information improves the image quality prediction rate. It is worth mentioning that combining both local (LBP map) and global (LBP histogram) features for computing the LBPSI provided the best performance in the proposed $L B P S I$ method. The proposed LBPSI improved the RMSE, PCC and SRC metrics by $1.1 \%, 1.7 \%$ and $1.4 \%$, respectively, compared to the results obtained based on the LBPSIWM .

TABLE II. THE RESULTS OBTAINED EMPLOYING THE PROPOSED METHOD ON THE ITESOFT DATASET [14] WITH DIFFERENT LBPSIS.

\begin{tabular}{lcccc}
\hline \hline Result & LBPSIM & LBPSIH & LBPSIWM & LBPSI \\
\hline RMSE & 0.110 & 0.095 & 0.097 & $\mathbf{0 . 0 8 8}$ \\
PCC & 0.888 & 0.919 & 0.914 & $\mathbf{0 . 9 3 1}$ \\
SRC & 0.877 & 0.905 & 0.905 & $\mathbf{0 . 9 1 9}$
\end{tabular}

TABLE III. THE RESULTS OBTAINED EMPLOYING THE PROPOSED METHOD ON THE ITESOFT DATASET [14] WITH DIFFERENT ARTIFACTS.

\begin{tabular}{|c|c|c|c|}
\hline Result ${ }^{\text {Artifact }}$ & JPEG & JPEG2000 & All images \\
\hline RMSE & 0.076 & 0.090 & 0.088 \\
\hline PCC & 0.941 & 0.933 & 0.931 \\
\hline SRC & 0.922 & 0.931 & 0.919 \\
\hline
\end{tabular}

Since, the ITESOFT dataset [14] is composed of images having two different types of distortions, the performance of the proposed $L B P S I$ with respect to different distortion types is demonstrated in Table III. The results shown in Table III indicate that the proposed LBPSI performed considerably well in relation to both distortion types. It can, however, be noted that the LBPSI performed slightly better on the images distorted by JPEG artifacts. A graphical representation of the objective quality assessment results obtained based on the proposed method in this research work against the subjective MHOS scores on the ITESOFT dataset [14] is provided in Fig. 3(a). From Fig. 3(a), it is clear that the results obtained from the proposed method and the subjective MHOS values are fairly correlated in a linear way. This linear correlation is an important property of the proposed method that can be modeled in a straightforward way.

As in the proposed LBPSI method there are a few parameters, which may impact on the results. We also provided an experimental study considering a range of values for the parameters $\mathrm{c}$ and $\alpha$. The results obtained from the proposed LBPSI based on different values of $c$ and $\alpha$ are shown in Tables IV and V. From Table IV, it can be concluded that the LBPSI method provided quite stable results when considering small values for parameter $c$. Hence, parameter $c$ was set to 0.0001 . Regarding parameter $\alpha$, the best results were obtained when parameter $\alpha$ was set to 0.30 (see Table V). Based on our experiments presented in Table $\mathrm{V}$, we noticed that a value between 0.25 and 0.35 for $\alpha$ could also provide the same results as obtained when considering $\alpha=030$.

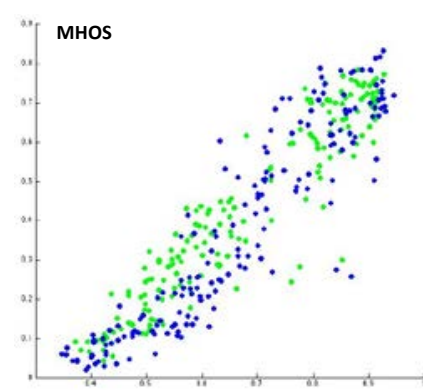

a) Proposed LBPSI

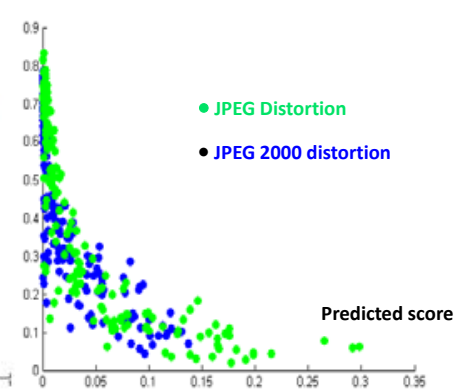

b) The MGMSD
Fig. 3. Scatter plots of the predicted quality scores based on a) the proposed LBPSI, and b) the MGMSD method against subjective MHOS scores on the ITESOFT dataset [14].

TABLE IV. THE RESULTS OBTAINED EMPLOYING THE PROPOSED METHOD ON THE ITESOFT DATASET [14] CONSIDERING DIFFERENT VALUES FOR $c$.

\begin{tabular}{lcccccccc}
\hline Result & C & \multirow{150}{*}{$\mathbf{1 0 0}$} & $\mathbf{1}$ & $\mathbf{0 . 0 1}$ & $\mathbf{0 . 0 0 1}$ & $\mathbf{0 . 0 0 0 1}$ & $\mathbf{0 . 0 0 0 0 1}$ \\
\hline RMSE & 0.091 & 0.091 & 0.088 & 0.088 & 0.088 & $\mathbf{0 . 0 8 8}$ & 0.088 \\
PCC & 0.925 & 0.926 & 0.930 & 0.931 & 0.931 & $\mathbf{0 . 9 3 1}$ & 0.931 \\
SRC & 0.911 & 0.912 & 0.918 & 0.919 & 0.919 & $\mathbf{0 . 9 1 9}$ & 0.919
\end{tabular}

TABLE V. THE RESULTS OBTAINED EMPLOYING THE PROPOSED METHOD ON THE ITESOFT DATASET [14] CONSIDERING DIFFERENT VALUES FOR $\boldsymbol{\alpha}$.

\begin{tabular}{lcccccccc}
\hline & $\alpha$ & $\mathbf{0 . 2 0}$ & $\mathbf{0 . 2 5}$ & $\mathbf{0 . 3 0}$ & $\mathbf{0 . 5 0}$ & $\mathbf{0 . 6 7}$ & $\mathbf{0 . 7 5}$ & $\mathbf{0 . 8 0}$ \\
\cline { 4 - 9 } Result & & & & & & & & \\
\hline RMSE & 0.088 & 0.088 & $\mathbf{0 . 0 8 8}$ & 0.089 & 0.091 & 0.092 & 0.092 \\
PCC & 0.930 & 0.931 & $\mathbf{0 . 9 3 1}$ & 0.929 & 0.925 & 0.924 & 0.923 \\
SRC & 0.918 & 0.919 & $\mathbf{0 . 9 1 9}$ & 0.917 & 0.913 & 0.911 & 0.910
\end{tabular}

TABLE VI. THE COMPARISON OF THE RESULTS OBTAINED EMPLOYING THE PROPOSED METHOD AND THE MGMSD [14] ON THE ITESOFT DATASET.

\begin{tabular}{lllcc}
\hline \hline Method & Metric & JPEG & JPEG2000 & All images \\
\hline \multirow{3}{*}{ MGMSD [14] } & RMSE & 0.097 & 0.090 & 0.096 \\
& PCC & 0.902 & $\mathbf{0 . 9 3 5}$ & 0.917 \\
& SRC & 0.895 & $\mathbf{0 . 9 3 4}$ & 0.916 \\
\hline \multirow{3}{*}{ Proposed LBPSI } & RMSE & $\mathbf{0 . 0 7 6}$ & $\mathbf{0 . 0 9 0}$ & $\mathbf{0 . 0 8 8}$ \\
& PCC & $\mathbf{0 . 9 4 1}$ & 0.933 & $\mathbf{0 . 9 3 1}$ \\
& SRC & $\mathbf{0 . 9 2 2}$ & 0.931 & $\mathbf{0 . 9 1 9}$
\end{tabular}

\section{Comparative analysis}

To have a fair comparison, initially, the results obtained from the proposed LBPSI method on the ITESOFT dataset are compared to the results obtained based on a recent DIQA method (MGMSD) presented in [14]. The results are shown in Table VI. From Table VI, it is evident that the proposed LBPSI method outperformed the MGMSD method [14] in all metrics. The highest improvement occurred for the PCC where a $1.4 \%$ (0.931-0.917) improvement was achieved. Regarding the results obtained based on different distortions, it is clear that a significant improvement (3.9\% for PCC) was achieved for the images distorted by JPEG artifacts. This improvement conveys that the LBP-based features can fairly characterize JPEG artifacts as is shown in the results of the LBPSI method. The scatter plots of the results obtained from the LBPSI and MGMSD methods shown in Fig. 3(a) and Fig. 3(b) also indicate that the results obtained from the LBPSI and the subjective MHOS values are fairly correlated in a linear way, whereas the results obtained from the 
MGMSD and the subjective MHOS values are exponentially correlated.

We further considered some state-of-the-art IQA methods [3, 4, 14] and computed their results on the ITESOFT dataset [14]. Compared to the four methods, which are considered as state-of-the-art technologies, the proposed LBPSI achieved/reached the best performance on the ITESOFT dataset as indicated in Table VIII.

To compare the performance of the proposed LBPSI method on natural images, the LIVE dataset [17] was further considered for experiments. The results obtained based on different LBPSI metrics proposed in this research work are shown in Table VII. The best performance was achieved based on the LBPSIWM. The LBPSIM provided reasonable results, whereas the $L B P S I H$ achieved a poor performance on natural images. The final LBPSI results were as good as the results obtained based on the $L B P S I W M$. The reason for the poor performance of the LBPSIH on the LIVE dataset might be the presence of five types of distortions in the LIVE dataset of which some could not be well characterized by the LBP histogram features. A comparison of the results obtained based on different IQA methods on the LIVE dataset [17] is presented in Table VIII. From the results obtained on the LIVE dataset, it can be concluded that the proposed LBPSI achieved competitive results compared to those obtained from some of the superior state-of-the-art IQA methods [3, 4, 14].

TABLE VII. THE RESULTS OBTAINED EMPLOYING THE PROPOSED METHOD ON THE LIVE DATASET [17] CONSIDERING DIFFERENT LBPSIS.

\begin{tabular}{|c|c|c|c|c|}
\hline Result $\quad$ Index & LBPSIM & LBPSIH & LBPSIWM & LBPSI \\
\hline RMSE & 11.89 & 17.15 & 9.34 & 9.53 \\
\hline PCC & 0.901 & 0.778 & 0.940 & 0.933 \\
\hline SRC & 0.903 & 0.779 & 0.938 & 0.935 \\
\hline
\end{tabular}

TABLE VIII. COMPARISON OF THE RESULTS OBTAINED FROM THE PROPOSED LBPSI AND RESULTS OF SOME STATE-OF-THE-ART METHODS.

\begin{tabular}{llccc}
\hline \hline Dataset & Method & RMSE & PCC & SRC \\
\hline \multirow{4}{*}{ LIVE [17] } & PSNR & 13.36 & 0.872 & 0.876 \\
& SSIM [3] & 8.95 & 0.945 & 0.948 \\
& GMSD [4] & $\mathbf{7 . 6 2}$ & $\mathbf{0 . 9 6 0}$ & $\mathbf{0 . 9 6 0}$ \\
& MGMSD [14] & 8.82 & 0.946 & 0.951 \\
& Proposed LBPSI & 9.34 & 0.940 & 0.938 \\
\hline \multirow{5}{*}{ ITESOFT } & PSNR & 0.147 & 0.791 & 0.796 \\
& SSIM [3] & 0.129 & 0.843 & 0.837 \\
& GMSD [4] & 0.122 & 0.860 & 0.839 \\
& MGMSD [14] & 0.096 & 0.917 & 0.916 \\
& Proposed LBPSI & $\mathbf{0 . 0 8 8}$ & $\mathbf{0 . 9 3 1}$ & $\mathbf{0 . 9 1 9}$
\end{tabular}

\section{CONCLUSIONS AND FUTURE WORK}

In this paper, a novel FR DIQA, based on the LBP texture features extracted at local and global levels, is proposed. The proposed LBPSI method performs well on document images. The results on natural scene images are also encouraging. The results obtained based on the weighted local LBP similarity map ( $L B P S I W M)$ demonstrated a significant improvement in automatically predicting image quality especially for document images. However, the best results were obtained based on the LBPSI when combining both local and global LBP similarity indices. These results support the assumption that the use of both local and global LBP texture features provides better DIQA. In future, we plan to study the use of other global and local features, especially applicable for document images, which may improve the objective DIQA metrics.

\section{REFERENCES}

[1] Z.Wang, A. C. Bovik, E. P. Simoncelli, "Structural approaches to image quality assessment," in Handbook of Image and Video Processing, pp. 961-974, Academic Press, $2^{\text {nd }}$ Ed., 2005.

[2] Z. Wang, A. C. Bovik, B. R. Sheikh, E. P. Simoncelli, "Image quality assessment: from error visibility to structural similarity,” IEEE Trans. on Image Processing, 13(4), pp. 600-612, 2004.

[3] L. Zhang, L. Zhang, X. Mou, D. Zhang, "FSIM: A feature similarity index for image quality assessment," IEEE Trans. on Image Processing, 20(8), pp. 2378-2386, 2011.

[4] W. Xue, L. Zhang, X. Mou, A. C. Bovik, "Gradient magnitude similarity deviation: A highly efficient perceptual image quality index,” IEEE Trans. on Image Processing, 23(2), pp. 684-695, 2014.

[5] A. Mittal, G. S. Muralidhar, J. Ghosh, A. C. Bovik, "Blind image quality assessment without human training using latent quality factors,” IEEE Signal Processing Letters, 19(2), pp. 75-78, 2012.

[6] P. Ye, J. Kumar, L. Kang, D. S. Doermann, "Unsupervised feature learning framework for no-reference image quality assessment," in Proc. of the CVPR, pp. 1098-1105, 2012.

[7] C. Hale, E. H. Barney Smith, "Human image preference and document degradation models," in Proc. of the $9^{\text {th }}$ ICDAR, pp. 257-261, 2007.

[8] L. Kang, P. Ye, Y. Li, D. Doermann, "A deep learning approach to document image quality assessment," in Proc. of the $22^{\text {nd }}$ ICIP, pp. 2570-2574, 2014.

[9] N. Nayef, J. M. Ogier, "Metric-based no-reference quality assessment of heterogeneous document images," In Proc. of SPIE 9402, Document Recognition and Retrieval (DRR) XXII, 94020L, 2015.

[10]P. Ye, D. Doermann, "Document image quality assessment: a brief survey," in Proc. of the $12^{\text {th }}$ ICDAR, pp. 723-727, 2013.

[11]H. Lu, A. C. Kot, Y. Q. Shi, "Distance-reciprocal distortion measure for binary document images," IEEE Signal Processing Letters, 11(2), pp. 228-231, 2004.

[12] T. Obafemi-Ajayi, G. Agam, "Character-based automated human perception quality assessment in document images," IEEE Trans. On SMC-Part A: Systems and Humans, 42(3), pp. 584-595, 2012.

[13] J. Kumar, F. Chen, D. Doermann, "Sharpness estimation for document and scene images," in Proc. of the 21 $1^{\text {st }}$ ICPR, pp. 3292-3295, 2012.

[14]A. Alaei, D. Conte, R. Raveaux, "Document Image Quality Assessment based on Improved Gradient Magnitude Similarity Deviation," in Proc. of the $13^{\text {th }}$ ICDAR, pp. 176-180, 2015.

[15] T. Ojala, M. Pietikainen, D. Harwood, "Performance Evaluation of Texture Measures with Classification Based on Kullback Discrimination of Distributions," in Proc. of the $12^{\text {th }}$ ICPR, pp. 582585, 1994.

[16]T. Ojala, M. Pietikainen, T. Maenpaa, "Multiresolution Gray-Scale and Rotation Invariant Texture Classification with Local Binary Patterns," IEEE Transactions on Pattern Analysis and Machine Intelligence, 24(7), pp. 971-987, 2002.

[17] H.R. Sheikh, K. Seshadrinathan, A.K. Moorthy, Z. Wang, A.C. Bovik, L.K. Cormack, "Image and video quality assessment research at LIVE,” http://live.ece.utexas.edu/research/quality.

[18] Z. Wang, and Q. Li, "Information content weighting for perceptual image quality assessment,” IEEE Trans. on Image Processing, 20(5), pp. 1185-1198, 2011.

[19]W. Xue, L. Zhang, X. Mou, "Learning without human scores for blind image quality assessment,” in Proc. of the CVPR, pp. 995-1002, 2013. 